\title{
The Lorentzian version of a theorem of Krust
}

\author{
Rafael López* \\ Departamento de Geometría y Topología \\ Universidad de Granada \\ 18071 Granada, Spain \\ rcamino@ugr.es
}

\begin{abstract}
In Lorentz-Minkowski space, we prove that the conjugate surface of a maximal graph over a convex domain is also a graph. We provide three proofs of this result that show a suitable correspondence between maximal surfaces in Lorentz-Minkowski space and minimal surfaces in Euclidean space.
\end{abstract}

Keywords: maximal surface, minimal surface, dual surface, conjugate surface AMS Subject Classification: 53A10, 53C42

\section{Introduction and statement of the result}

Romain Krust proved the following result about minimal graphs in Euclidean space $\mathbb{E}^{3}$ : see $[5$, p. 188] and [7, p. 33].

Theorem 1.1. If an embedded minimal surface $X: B \rightarrow \mathbb{E}^{3}, B=\{w \in \mathbb{C}:|w|<$ $1\}$, can be written as a graph over a convex domain in a plane, then the conjugate surface $X^{*}: B \rightarrow \mathbb{E}^{3}$ is a graph.

\footnotetext{
*Partially supported by the grant no. MTM2017-89677-P, MINECO/AEI/FEDER, UE
} 
In this paper, we extend this result for maximal surfaces in Lorentz-Minkowski space $\mathbb{L}^{3}$. An immersion of a surface in $\mathbb{L}^{3}$ is called spacelike if the induced metric on the surface is Riemannian. A maximal surface in $\mathbb{L}^{3}$ is a spacelike surface with zero mean curvature at every point. From the variational viewpoint, maximal surfaces locally represent a maximum for the area integral. The Lorentzian version of the Krust's theorem is formally the same, except that we need to precise the causal character of the plane with respect to which the surface is a graph.

Theorem 1.2. If an embedded maximal surface $X: B \rightarrow \mathbb{L}^{3}, B=\{w \in \mathbb{C}:|w|<$ $1\}$, can be written as a graph over a convex domain in a spacelike plane, then the conjugate surface $X^{*}: B \rightarrow \mathbb{L}^{3}$ is a graph.

Although this result may be expected, there are differences in the theory of minimal surfaces in $\mathbb{E}^{3}$ and maximal surfaces in $\mathbb{L}^{3}$. A clear example is the Bernstein theorem. In $\mathbb{L}^{3}$, the only maximal entire graphs are spacelike planes $([4])$, which is the Lorentzian version of the classical Bernstein theorem in Euclidean space. However, the result holds for arbitrary dimensions, that is, for maximal hypersurfaces in $\mathbb{L}^{n}$, in contrast with the Euclidean version, where the Bernstein theorem only is valid for minimal hypersurfaces of $\mathbb{E}^{n}$ with $n \leq 7$ ([2]).

The goal of this paper is to provide three different approaches of the proof of Theorem 1.2. A first proof follows the same steps as in Euclidean space by means of the Weierstrass representation of a maximal surface (Section 3). The second proof makes use of a clever geometric idea due to F. Martín in [15] (Section 4). Finally, the third proof uses a duality correspondence between maximal surfaces of $\mathbb{L}^{3}$ and minimal surface of $\mathbb{E}^{3}$ (Section 5).

\section{Preliminaries}

In this section we fix the terminology and notation. The Lorentz-Minkowski 3dimensional space $\mathbb{L}^{3}$ is the vector space $\mathbb{R}^{3}$ with canonical coordinates $\left(x_{1}, x_{2}, x_{3}\right)$ and endowed with the metric $\langle\rangle=,d x_{1}^{2}+d x_{2}^{2}-d x_{3}^{2}$. A vector $v \in \mathbb{R}^{3}$ is spacelike, timelike or lightlike if $\langle v, v\rangle$ is positive, negative or zero, respectively. We refer the reader to [12] for general definitions of $\mathbb{L}^{3}$. In order to distinguish the Euclidean space from the Lorentzian space, we denote the Euclidean space by $\mathbb{E}^{3}$, that is, $\mathbb{R}^{3}$ with the Euclidean metric $\langle,\rangle_{0}=d x_{1}^{2}+d x_{2}^{2}+d x_{3}^{2}$. 
The spacelike condition for a surface in $\mathbb{L}^{3}$ is a strong property. For example, any spacelike (connected) surface $X: M \rightarrow \mathbb{L}^{3}$ is orientable. This is due to the fact the two timelike vectors are not orthogonal. Indeed, the orthogonal subspace to each tangent plane $T_{p} M$ is timelike. Thus, if $N$ is a local orientation on $M, N(p)$ is a unit timelike vector and if $e_{3}=(0,0,1)$, then $\left|\left\langle N(p), e_{3}\right\rangle\right| \geq 1$ for every $p \in M$. Hence, by connectedness, it is possible to choose a global orientation $N$ on $M$ such that $\left\langle N, e_{3}\right\rangle \leq-1$ globally in $M$ (or $\left\langle N, e_{3}\right\rangle \geq 1$ in $M$ ), which proves the orientability of $M$. In this paper, we will choose the orientation on a spacelike surface such that $\left\langle N, e_{3}\right\rangle \leq-1$ on $M$.

Other consequence of the spacelike condition is when we consider spacelike graphs in $\mathbb{L}^{3}$. It is known that any surface $X: M \rightarrow \mathbb{R}^{3}$ (without any induced metric) is locally the graph over one of the three coordinate planes of $\mathbb{R}^{3}$. However, if $X: M \rightarrow \mathbb{L}^{3}$ is a spacelike immersion, then we can assure that the surface is locally a graph on the $x_{1} x_{2}$-plane. Indeed, consider the orthogonal projection onto the $x_{1} x_{2}$-plane, which we identify with $\mathbb{R}^{2}=\mathbb{C}$ :

$$
\pi: \mathbb{R}^{3} \rightarrow \mathbb{R}^{2}, \quad \pi\left(x_{1}, x_{2}, x_{3}\right)=\left(x_{1}, x_{2}\right) .
$$

Define the map $\tilde{x}=\pi \circ X: M \rightarrow \mathbb{R}^{2}$. The differential $(d \tilde{x})_{p}$ at $p$ is $(d \tilde{x})_{p}(v)=\left(v_{1}, v_{2}\right)$, $v=\left(v_{1}, v_{2}, v_{3}\right) \in T_{p} M$. Then

$$
\left|(d \tilde{x})_{p}(v)\right|^{2}=v_{1}^{2}+v_{2}^{2} \geq v_{1}^{2}+v_{2}^{2}-v_{3}^{2}=\langle v, v\rangle>0
$$

for any nonzero tangent vector $v$, and consequently, $(d \tilde{x})_{p}$ is injective. This proves that $\tilde{x}$ is a local diffeomorphism.

In the classical theory of minimal surfaces in Euclidean space, it is an issue to determine when a minimal surface is a minimal graph. One of the first results was obtained by Radó proving that if $X: B \rightarrow \mathbb{E}^{3}$ is a compact minimal disk and $X(\partial B)$ can be orthogonally projected one-to-one onto a planar convex closed curve $\Gamma$, then $X(B)$ is a minimal graph over the convex planar domain bounded by $\Gamma([16])$. For spacelike surfaces, without assuming any assumption on its mean curvature, the result goes beyond.

Proposition 2.1. Let $\Gamma \subset \mathbb{R}^{3}$ be a simple closed curve and let $X: M \rightarrow \mathbb{L}^{3}$ be a compact spacelike surface such that $X: \partial M \rightarrow \Gamma$ is a diffeomorphism. If there exists a spacelike plane $P$ such that the orthogonal projection of $\Gamma$ on $P$ is a simple closed curve, then $X(M)$ is a spacelike graph on some domain of $P$. In particular, a compact spacelike surface spanning a simple closed planar curve is a graph. 
Proof. After a rigid motion, we can assume that $P$ is the plane of equation $x_{3}=0$. By (1), we know that $\tilde{x}=\pi \circ X: \operatorname{int}(M) \rightarrow \mathbb{R}^{2}$ is a local diffeomorphism and, therefore, it is an open map. Let $\Omega=\tilde{x}(\operatorname{int}(M)) \subset \mathbb{R}^{2}$, which is an open subset in $\mathbb{R}^{2}$, and let $\Omega^{\prime}$ be the planar domain bounded by the plane simple closed curve $\Gamma^{\prime}=\pi(\Gamma)=\tilde{x}(\partial M)$.

1. Claim: $\partial \tilde{x}(M) \subset \Gamma^{\prime}$.

Since $M$ is compact, for any $q \in \partial \tilde{x}(M)$ there exists $p \in M$ such that $\tilde{x}(p)=q$. We show that $p \in \partial M$. On the contrary, $p \in \operatorname{int}(M)$ and there is an open neighborhood $U_{p}$ of $p$ in $\operatorname{int}(M)$ and an open neighborhood $V_{q}$ of $q$ in $\Omega$ such that $\tilde{x}: U_{p} \rightarrow V_{q}$ is a diffeomorphism. This implies that $q \in \Omega$, contradicting that $q$ is a boundary point of $\tilde{x}(M)$.

2. Claim: $\Omega=\Omega^{\prime}$.

If there exists a point in $\Omega$ which is not in $\Omega^{\prime}$ and since $\Omega$ is bounded, there are points in $\partial \Omega$ outside $\Omega^{\prime}$, which is impossible. Analogously, if there is a point in $\Omega^{\prime}$ which is not in $\Omega$, there are points in $\partial \Omega$ inside $\Omega^{\prime}$, which is not possible again.

As a consequence, $\tilde{x}: M \rightarrow \bar{\Omega}$ is a local diffeomorphism, and the compactness of $M$ implies that $\tilde{x}$ is a covering map. Since $\bar{\Omega}$ is simply connected, the map $\tilde{x}$ must be a global diffeomorphism.

Hence, letting $F=\tilde{x}^{-1}$ we conclude that $x \circ F$ is the graph determined by the function $f=x_{3} \circ F$.

For the last statement, assume that the boundary $\Gamma$ of the surface is planar. Because any curve contained in a spacelike surface is spacelike, the curve $\Gamma$ is spacelike. Since $\Gamma$ is a closed curve, the plane containing $\Gamma$ must be spacelike ([12]). This proves that $\Gamma$ is contained in a spacelike plane, and the result applies.

Minimal surfaces in $\mathbb{E}^{3}$ and maximal surfaces in $\mathbb{L}^{3}$ share some properties. For example, maximal surfaces admit a Weierstrass representation as it occurs for minimal surfaces and that we now explain ([8]). Let $M$ be an orientable surface and consider isothermal parameters on $M$ which induce a conformal structure on $M$. Let $X: M \rightarrow \mathbb{L}^{3}$ be a spacelike conformal immersion such that the mean curvature 
vanishes at every point of $M$, that is, $X$ is a maximal surface. If $N$ is the Gauss map of $X$, and because $\left\langle N, e_{3}\right\rangle \leq-1$, then $N$ is a map

$$
N: M \rightarrow \mathbb{H}^{2}:=\left\{x=\left(x_{1}, x_{2}, x_{3}\right) \in \mathbb{R}^{3}:\langle x, x\rangle=-1, x_{3} \geq 1\right\} .
$$

If $\overline{\mathbb{C}}=\mathbb{C} \cup\{\infty\}$ is the extended complex plane and $\mu: \mathbb{H}^{2} \rightarrow \overline{\mathbb{C}} \backslash\{|z|=1\}$ is the stereographic projection from the North pole $(0,0,1) \in \mathbb{H}^{2}$, the Gauss map $N$ is viewed as a meromorphic function $g: M \rightarrow \overline{\mathbb{C}}$, with $g=\mu \circ N$.

Define a $\mathbb{C}^{3}$-valued holomorphic 1 -form on $M$ by $\Psi=2 d X=2 X_{z} d z$, where $z$ is a complex coordinate of $M$. There is a holomorphic 1-form $\eta$ on $M$ such that the 1 -forms

$$
\Psi_{1}=\frac{1}{2}\left(1+g^{2}\right) \eta, \quad \Psi_{2}=\frac{i}{2}\left(1-g^{2}\right) \eta, \quad \Psi_{3}=-g \eta
$$

are holomorphic on $M$ without common zeroes and they have no real periods. If $z_{0} \in M$ is a base point, then the immersion $X$ is determined by

$$
X(z)=X\left(z_{0}\right)+\operatorname{Re} \int_{z_{0}}^{z} \Psi, \quad \Psi=\left(\Psi_{1}, \Psi_{2}, \Psi_{3}\right) .
$$

We say that $(M, g, \eta)$ (or $(M, \Psi))$ are the Weierstrass data of $X$ and (2) is the Weierstrass representation of $X$. The complex curve $\Psi$ associate to $X$ is isotropic in the sense that $\langle\Psi, \Psi\rangle=\Psi_{1}^{2}+\Psi_{2}^{2}-\Psi_{3}^{2}=0$, where $\langle$,$\rangle denotes the complexification$ of the Lorentzian metric.

Suppose that $X$ is defined on a simply connected domain $\Omega$ of $\mathbb{R}^{2}=\mathbb{C}$. The conjugate surface $X^{*}: \Omega \rightarrow \mathbb{L}^{3}$ is defined on $\Omega$ as solution of the Cauchy-Riemann equations

$$
X_{u}^{*}=-X_{v}, \quad X_{v}^{*}=X_{u}
$$

in $\Omega$, where $z=u+i v \in \Omega, i=\sqrt{-1}$. Then $X^{*}: \Omega \rightarrow \mathbb{L}^{3}$ is a conformal spacelike immersion which is also a maximal surface. The map $X^{*}$ is nothing that the harmonic conjugate of $X$, that is, the map $\tilde{X}=X+i X^{*}: \Omega \rightarrow \mathbb{C}^{3}$ is holomorphic and the complex derivative of $\tilde{X}$ is $\tilde{X}_{z}=X_{u}+i X_{u}^{*}=X_{u}-i X_{v}=\Psi / 2$. In particular, up to a constant, $X^{*}(z)=\operatorname{Im} \int^{z} \Psi d z$. Moreover

$$
d X^{*}=-d X \circ J
$$

where $J$ is the rotation of 90 degrees on all tangent planes induced by the orientation on $\Omega$. More precisely, if $\left\{\partial_{u}, \partial_{v}\right\}$ is the oriented basis in the tangent plane determined by the conformal parameter $z$, then

$$
J\left(\partial_{u}\right)=\partial_{v}, \quad J\left(\partial_{v}\right)=-\partial_{u} .
$$


The conjugate surface $X^{*}$ is isometric to the initial surface $X$ and both surfaces have the same Gauss map at corresponding points. The isotropic curve of $X^{*}$ is

$$
\Psi^{*}=2 d X^{*}=2 X_{z}^{*} d z=2\left(X_{u}^{*}-i X_{v}^{*}\right) d z=-2 i\left(X_{u}-i X_{v}\right) d z=-i \Psi,
$$

and the Weierstrass data of $X^{*}$ is $(\Omega, g,-i \eta)$.

\section{First proof: using the Weierstrass representa- tion}

In this section we follow the same steps that the original idea of Krust by means of the Weierstrass representation formula for a maximal surface. Firstly, we change the expression (2) by defining a meromorphic function $h$ such that $d h=g \eta$. Then (2) is now

$$
X(w)=X\left(w_{0}\right)+\operatorname{Re} \int_{w_{0}}^{w}\left(\frac{1}{2}\left(\frac{1}{g}+g\right), \frac{i}{2}\left(\frac{1}{g}-g\right),-1\right) d h .
$$

After a rigid motion of $\mathbb{L}^{3}$, we can assume that $X$ is graph on a convex domain $D$ of the plane of equation $x_{3}=0$, which we identify with $\mathbb{R}^{2}=\mathbb{C}$. By our choice of orientation for a spacelike surface explained in Section 2, the Gauss map $N$ points upwards in $B$. Because the inverse of the stereographic projection is

$$
\mu^{-1}(z)=\left(\frac{-2 \operatorname{Re} z}{|z|^{2}-1}, \frac{-2 \operatorname{Im} z}{|z|^{2}-1}, \frac{|z|^{2}+1}{|z|^{2}-1}\right)
$$

and $N=\mu^{-1} \circ g$, we deduce that $|g(z)|>1$ in $B$.

Without loss of generality, we assume $w_{0}=0$ and $X\left(w_{0}\right)=0$ in (5). Let us introduce the following notation:

$$
\sigma(w)=-\int_{0}^{w} \frac{g}{2} d h, \quad \tau(w)=\int_{0}^{w} \frac{1}{2 g} d h .
$$

Using $\sigma$ and $\tau$ together (5), the orthogonal projection of $X(B)$ is

$$
\begin{aligned}
\pi \circ X(w) & =\operatorname{Re} \int_{0}^{w}\left(\frac{1}{2}\left(\frac{1}{g}+g\right), \frac{i}{2}\left(\frac{1}{g}-g\right)\right) d h \\
& =\operatorname{Re}(\tau-\sigma+i(t \tau+\sigma))=\bar{\tau}-\sigma .
\end{aligned}
$$


Analogously, and because $X^{*}(w)=\operatorname{Im} \int_{0}^{w} \Psi$, we have

$$
\begin{aligned}
\pi \circ X^{*}(w) & =\operatorname{Im} \int_{0}^{w}\left(\frac{1}{2}\left(\frac{1}{g}+g\right), \frac{i}{2}\left(\frac{1}{g}-g\right)\right) d h \\
& =\operatorname{Im}(\tau-\sigma+i(\tau+\sigma))=i(\bar{\tau}+\sigma) .
\end{aligned}
$$

Claim: If $w_{1} \neq w_{2}, w_{1}, w_{2} \in B$, then $\pi \circ X^{*}\left(w_{1}\right) \neq \pi \circ X^{*}\left(w_{2}\right)$.

Let $p_{i}=\pi \circ X\left(w_{i}\right)$ and $q_{i}=\pi \circ X^{*}\left(w_{i}\right), i=1,2$. Since $X(B)$ is a graph on the convex domain $D=\pi \circ X(B)$, there is a segment in $D$ connecting $p_{1}$ with $p_{2}$. We parametrize this segment by

$$
\gamma(t):[0,1] \rightarrow D, \quad \gamma(t)=(1-t) p_{1}+t p_{2}
$$

and let $\beta:[0,1] \rightarrow B$ be a curve in the unit disk $B$ such that $X \circ \beta=\pi_{\mid X(B)}^{-1} \circ \gamma$. Then

$$
p_{2}-p_{1}=\gamma(1)-\gamma(0)=\gamma^{\prime}(t)
$$

Using (6),

$$
\begin{aligned}
p_{2}-p_{1} & =d(\pi \circ X) \beta^{\prime}(t)=d(\bar{\tau}-\sigma) \beta^{\prime}(t) \\
& =\left.\left(\overline{\frac{h^{\prime}(w)}{2 g(w)}}-\frac{g(w) h^{\prime}(w)}{2}\right)\right|_{w=\beta(t)} \beta^{\prime}(t)
\end{aligned}
$$

Similarly, taking into account (7), we obtain

$$
\begin{aligned}
q_{2}-q_{1} & =\int_{\gamma} d\left(\pi \circ X^{*}\right) \beta^{\prime}(t)=\int_{\gamma} i d(\bar{\tau}+\sigma) \beta^{\prime}(t) \\
& =\left.\int_{\gamma} i\left(\overline{\frac{h^{\prime}(w)}{2 g(w)}}+\frac{g(w) h^{\prime}(w)}{2}\right)\right|_{w=\beta(t)} \beta^{\prime}(t)
\end{aligned}
$$

We multiply $p_{2}-p_{1}$ and $i\left(q_{2}-q_{1}\right)$ with the Euclidean scalar product $\langle,\rangle_{0}$ of $\mathbb{R}^{2}$. Recall that in complex notation, $\left\langle v_{1}, v_{2}\right\rangle_{0}=\operatorname{Re}\left(v_{1} \overline{v_{2}}\right), v_{1}, v_{2} \in \mathbb{R}^{2}$. Thus (8) and (9) 
imply

$$
\begin{aligned}
\left\langle p_{2}-p_{1}, i\left(q_{2}-q_{1}\right)\right\rangle & =\operatorname{Re}\left(\left(p_{2}-p_{1} \overline{\left(i\left(q_{2}-q_{1}\right)\right)}\right)\right. \\
& =-\left.\int_{0}^{1} \operatorname{Re}\left(\overline{\frac{h^{\prime}(w)}{2 g(w)}}-\frac{g(w) h^{\prime}(w)}{2}\right)\left(\frac{h^{\prime}(w)}{2 g(w)}+\frac{\overline{g(w) h^{\prime}(w)}}{2}\right)\right|_{w=\beta(t)}\left|\beta^{\prime}(t)\right|^{2} \\
& =-\left.\int_{0}^{1} \frac{\left|\beta^{\prime}(t)\right|^{2}}{4}\left(\frac{1}{|g|^{2}}-|g|^{2}\right)\right|_{w=\beta(t)}>0 .
\end{aligned}
$$

Because $p_{1} \neq p_{2}$, we conclude $q_{1} \neq q_{2}$. This proves that the orthogonal projection $\pi: X^{*}(B) \rightarrow \mathbb{R}^{2}$ is injective, hence $X^{*}(B)$ is a graph.

\section{Second proof: a geometric approach}

In this proof, we begin as in the above section and we employ the notation that appeared there. Without loss of generality, we assume that $X(B)$ is a graph on the $x_{1} x_{2}$-plane of $\mathbb{R}^{3}$, which we identify with $\mathbb{R}^{2}$ again.

Claim: If $w_{1} \neq w_{2}, w_{1}, w_{2} \in B$, then $\pi \circ X^{*}\left(w_{1}\right) \neq \pi \circ X^{*}\left(w_{2}\right)$.

Recall that $\gamma$ is the segment in $D$ that connects $p_{1}$ with $p_{2}$. Let $\Pi$ be the plane containing $\gamma$ and orthogonal to $\mathbb{R}^{2}$. Let $\vec{a}=\left(a_{1}, a_{2}, 0\right) \in \mathbb{L}^{3}$ be a unit vector orthogonal to $\Pi$. Define the curve $\alpha:[0,1] \rightarrow \mathbb{L}^{3}$ by $\alpha(t)=X(\beta(t))$, which connects $X\left(w_{1}\right)$ with $X\left(w_{2}\right)$. Denote $\alpha^{*}=X^{*} \circ \beta$ the conjugate curve of $\alpha$ connecting $X^{*}\left(w_{1}\right)$ with $X^{*}\left(w_{2}\right)$.

If we write $\beta^{\prime}(t)=u(t) \partial_{u}(t)+v(t) \partial_{v}(t)$, where $\left\{\partial_{u}, \partial_{v}\right\}$ is an oriented basis at each tangent plane of the $z$-plane, by $(3)$, we obtain

$$
\begin{aligned}
\alpha^{* \prime}(t) & =\left(d X^{*}\right)\left(\beta^{\prime}(t)\right)=-(d X)\left(-v(t) \partial_{u}(t)+u(t) \partial_{v}(t)\right) . \\
& =v(t) X_{u}(t)-u(t) X_{v}(t)
\end{aligned}
$$

On the other hand, if $N=\lambda\left(X_{u} \times X_{v}\right)$ is the Gauss map of $X, \lambda>0$, where $\times$ is the Lorentzian vector product in $\mathbb{L}^{3}$, and because $X$ is conformal, we have $N \times X_{u}=-X_{v}$ and $N \times X_{v}=X_{u}$. then

$$
N(\alpha(t)) \times \alpha^{\prime}(t)=-u(t) X_{v}(t)+v(t) X_{u}(t)=\alpha^{* \prime}(t) .
$$


Since $\alpha$ is contained in the plane $\Pi$, the vector $\nu(t)=N(\alpha(t)) \times \alpha^{\prime}(t)$ does not belong to the plane $\Pi$, hence $\nu(t)$ satisfies $\langle\nu(t), \vec{a}\rangle \neq 0$ for every $t \in[0,1]$. Since $\vec{a}$ is a horizontal vector, $\langle\nu(t), \vec{a}\rangle=\langle\nu(t), \vec{a}\rangle_{0}$. Without loss of generality, we assume $\langle\nu(t), \vec{a}\rangle>0$ in $[0,1]$. In particular, $\langle\pi \circ \nu(t), \vec{a}\rangle>0$ in $[0,1]$ because $\nu(t) \notin \Pi$ for every $t \in[0,1]$. Then

$$
\begin{aligned}
\left\langle q_{2}-q_{1}, \vec{a}\right\rangle & =\left\langle\int_{0}^{1}\left(\pi \circ \alpha^{*}\right)^{\prime}(t), \vec{a}\right\rangle d t=\left\langle\int_{0}^{1} \pi \circ \nu(t), \vec{a}\right\rangle d t \\
& =\int_{0}^{1}\langle\pi \circ \nu(t), \vec{a}\rangle d t>0 .
\end{aligned}
$$

Again, we conclude $q_{1} \neq q_{2}$. Thus $\pi: X^{*}(B) \rightarrow \mathbb{R}^{2}$ is injective, hence $X^{*}(B)$ is a graph.

\section{Third proof: using duality}

Between minimal surfaces and maximal surfaces there is a correspondence, called duality, that assigns to each minimal surface in $\mathbb{E}^{3}$ a maximal surface in $\mathbb{L}^{3}$ and viceversa (see $[10,11]$ for generalizations in other ambient spaces). It was Calabi the first who realized of this correspondence when the surfaces are expressed as graphs on simply connected domains ([3]). Indeed, assume that $S$ is a minimal graph in $\mathbb{E}^{3}$ of a function $f: \Omega \subset \mathbb{R}^{2} \rightarrow \mathbb{R}$ defined in a simply connected domain $\Omega$. Then the minimality of $S$ is equivalent to

$$
\operatorname{div}\left(\frac{D f}{\sqrt{1+|D f|^{2}}}\right)=0 .
$$

Since $\Omega$ is simply connected, there exists a solution $f^{b}: \Omega \rightarrow \mathbb{R}$ of the equation

$$
D f^{b}=\frac{\left(-f_{y}, f_{x}\right)}{\sqrt{1+|D f|^{2}}} .
$$

Moreover, $f^{b}$ satisfies $\left|D f^{b}\right|<1$ in $\Omega$, that is, the graph $S^{b}$ of $f^{b}$, viewed in the Lorentz-Minkowski space $\mathbb{L}^{3}$, is spacelike. On the other hand, it is immediate that

$$
\operatorname{div}\left(\frac{D f^{b}}{\sqrt{1-\left|D f^{b}\right|^{2}}}\right)=0
$$


which is equivalent to say that the mean curvature of $S^{b}$ vanishes identically. Thus $S^{b}$ is a maximal surface which is called the dual surface of $S$. We say that this duality is by graphs because the arguments are local. It is immediate a reverse process starting from a maximal graph $S$ in $\mathbb{L}^{3}$ and obtaining a minimal surface $S^{\sharp}$ in $\mathbb{E}^{3}$, called the dual surface of $S$.

On the other hand, a similar correspondence appeared in $[6,14]$, where the duality is defined in terms of the isotropic curve that determines the surface. Exactly, let $X: \Omega \subset \mathbb{C} \rightarrow \mathbb{E}^{3}, X=X(z)$, be a conformal minimal surface defined on a simply connected domain $\Omega$ and let $\Phi: \Omega \rightarrow \mathbb{C}^{3}$ be the isotropic curve such that $\Phi(z)=$ $2 X_{z} d z=\left(\Phi_{1}, \Phi_{2}, \Phi_{3}\right)$. Then $\langle\Phi, \Phi\rangle_{0}=0$, where $\langle,\rangle_{0}$ is the complexification in $\mathbb{C}^{3}$ of the Euclidean metric. We consider the 1 -form in $\mathbb{C}^{3}$ defined by $\Psi=\left(\Phi_{1}, \Phi_{2},-i \Phi_{3}\right)$. It is immediate that $\langle\Psi, \Psi\rangle=0$. The dual surface of $X$ is the maximal surface $X^{b}: \Omega \rightarrow \mathbb{L}^{3}$ defined by $X^{b}(z)=\operatorname{Re} \int^{z} \Psi(z)$. The converse process is similar. If $X: \Omega \rightarrow \mathbb{L}^{3}$ is a conformal maximal surface and $\Psi=\left(\Psi_{1}, \Psi_{2}, \Psi_{3}\right)$ is its isotropic curve, then $\Phi=\left(\Psi_{1}, \Psi_{2}, i \Psi_{3}\right)$ is the isotropic curve of a minimal surface in $\mathbb{E}^{3}$ by means of $X^{\sharp}(z)=\operatorname{Re} \int^{z} \Phi(z)$, which is called the dual surface of $X$. Furthermore, and up to translations of the ambient space, we have $M=\left(M^{\sharp}\right)^{b}$. If Min and Max denote the family of minimal surfaces of $\mathbb{E}^{3}$ and the maximal surfaces of $\mathbb{L}^{3}$, respectively, the duality is given by the two maps

$$
b: \operatorname{Min} \rightarrow \operatorname{Max}, \quad \sharp: \operatorname{Max} \rightarrow \operatorname{Min}
$$

with the property that $b \circ \sharp$ and $\sharp \circ b$ are the identities in Max and Min respectively.

It is important to remark that both definitions of duality coincide. This was proved by Lee and the key of this equivalence is the existence of a simultaneous conformal coordinates for a minimal graph and its dual maximal graph.

Proposition 5.1 ([9]). Up to a translation, the dual surface of a minimal (resp. maximal) graph over a simply connected domain of $\mathbb{R}^{2}$ coincides with the dual surface obtained by the correspondence b (resp. $\sharp$ ).

Other ingredient that we need in the proof of Theorem 1.2 is the behavior of duality by conjugations of minimal surfaces and maximal surfaces.

Lemma 5.2. Up to translations of the ambient space, the duality and the conjugation processes are commutative, that is, $\left(M^{b}\right)^{*}=\left(M^{*}\right)^{b}$ and $\left(M^{\sharp}\right)^{*}=\left(M^{*}\right)^{\sharp}$. 
Proof. We only prove the first identity because the other one is analogous. Let $X: M \rightarrow \mathbb{E}^{3}$ be a minimal surface and let $\Phi=\left(\Phi_{1}, \Phi_{2}, \Phi_{3}\right)$ be its isotropic curve. The isotropic curve of $X^{b}$ is $\left(\Phi_{1}, \Phi_{2},-i \Phi_{3}\right)$. By (4), the isotropic curve of $\left(X^{b}\right)^{*}$ is $-i\left(\Phi_{1}, \Phi_{2},-i \Phi_{3}\right)=\left(-i \Phi_{1},-i \Phi_{2},-\Phi_{3}\right)$.

On the other hand, we know that the isotropic curve $\Phi^{*}$ of $X^{*}: M \rightarrow \mathbb{E}^{3}$ is $\Phi^{*}=-i \Phi=-i\left(\Phi_{1}, \Phi_{2}, \Phi_{3}\right)=\left(-i \Phi_{1},-i \Phi_{2},-i \Phi_{3}\right)$. Then the isotropic curve of $\left(X^{*}\right)^{b}$ is $\left(-i \Phi_{1},-i \Phi_{2},-\Phi_{3}\right)$, which coincides with the one of $\left(X^{b}\right)^{*}$, proving the result.

We now present the third proof of Theorem 1.2. By using the dual correspondence, we carry the proof in a problem in Euclidean space $\mathbb{E}^{3}$. Then we use Theorem 1.1, and finally we come back to $\mathbb{L}^{3}$ by duality again. A similar idea was used by Alías and Palmer to prove the equivalence of the Bernstein theorem in $\mathbb{E}^{3}$ and $\mathbb{L}^{3}([1])$.

Let $X: B \rightarrow \mathbb{L}^{3}$ be a maximal graph on a convex domain $\Omega$ of a spacelike plane $P$. After a rigid motion, we assume that $P$ is the $x_{1} x_{2}$-plane, which we identify with $\mathbb{R}^{2}$. Let $X^{\sharp}: B \rightarrow \mathbb{E}^{3}$ be its dual surface (as a minimal graph). Since $\Omega$ is simply connected, we know by Proposition 5.1 that $X^{\sharp}(B)$ is a minimal graph on the same domain $\Omega$. Because $\Omega$ is convex, the (Euclidean) Krust's Theorem 1.1 asserts that the dual surface $\left(X^{\sharp}\right)^{*}: B \rightarrow \mathbb{E}^{3}$ is a minimal graph on some domain $\widetilde{\Omega} \subset \mathbb{R}^{2}$. Because $\left(X^{\sharp}\right)^{*}$ is an embedding and $B$ is the unit ball, the domain $\widetilde{\Omega}$ is simply connected. Using Proposition 5.1 again, the dual surface of $\left(X^{\sharp}\right)^{*}$, namely, $\left(\left(X^{\sharp}\right)^{*}\right)^{b}$, is a maximal graph on the same domain $\widetilde{\Omega}$. Finally, by Lemma 5.2 ,

$$
\left(\left(X^{\sharp}\right)^{*}\right)^{b}=\left(\left(X^{\sharp}\right)^{b}\right)^{*}=X^{*},
$$

proving that $X^{*}$ is a maximal graph. This concludes the proof.

\section{References}

[1] Alías, J. L., Palmer, B.: A duality result between the minimal surface equation and the maximal surface equation. An. Acad. Bras. Cienc. 73, 161-164 (2001)

[2] Bombieri, E., De Giorgi, E., Giusti, E.: Minimal cones and the Bernstein problem. Invent. Math. 7, 243-268 (1969) 
[3] Calabi, E.: Examples of Bernstein problems for some nonlinear equations. Proc. Symp. Pure Math. 15, 223-230 (1970)

[4] Cheng, S. Y., Yau, S. T.: Maximal space-like hypersurfaces in the LorentzMinkowski spaces. Ann. of Math. (2) 104, 407-419 (1976)

[5] Dierkes, U., Hildebrandt, S., Ster, A. K., Wohlrab, O.: Minimal Surfaces I: Boundary Value Problems. Grundlehren der Mathematischen Wissenschaften, Springer, 1992.

[6] Gu, C. H.: The extremal surfaces in the 3-dimensional Minkowski space. Acta Math. Sin. New Ser. 1, 173-180 (1985)

[7] Karcher, H.: Construction of minimal surfaces. Surveys in Geometry, 1-96, University of Tokyo, 1989 (also: Lecture Notes No. 12, SFB256, Bonn, 1989. Available: http://www.math.uni-bonn.de/people/karcher/karcherTokyo.pdf).

[8] Kobayashi, O.: Maximal surfaces in the 3-dimensional Minkowski space $L^{3}$. Tokyo J. Math. 6, 297-309 (1983)

[9] Lee, H.: Extension of the duality between minimal surfaces and maximal surfaces. Geom. Dedicata, 151, 373-386 (2011)

[10] Lee, H.: Minimal surface systems, maximal surface systems and special Lagrangian equations. Trans. Amer. Math. Soc. 365, 3775-3797 (2013)

[11] Lee, H., Manzano, J. M.: Generalized Calabi correspondence and complete spacelike surfaces. Asian J. Math. 23, 35-48 (2019)

[12] López, R.: Differential geometry of curves and surfaces in Lorentz-Minkowski space. Int. Electron. J. Geom. 7, 44-107 (2014)

[13] López, R., Kaya, S.: On the duality between rotational minimal surfaces and maximal surfaces. J. Math. Anal. Appl. 458, 345-360 (2018)

[14] López, F. J., López, R., Souam, R.: Maximal surfaces of Riemann type in Lorentz-Minkowski space $L^{3}$. Michigan Math. J. 47, 469-497 (2000)

[15] Martín, F., Reyes, C.: An extension of Krust's theorem for minimal surfaces. In: Florentino García Santos: in memoriam, 115-117, Ed. Univ. Granada, Granada, 2011. 
[16] Radó, T.: Contributions to the theory of minimal surfaces. Acta Litt. Scient. Univ. Szeged, 6, 1-20 (1932) 